\title{
ACE Inhibitor Benefit to Kidney and Cardiovascular Outcomes for Patients with Non-Dialysis Chronic Kidney Disease Stages 3-5: A Network Meta-Analysis of Randomised Clinical Trials
}

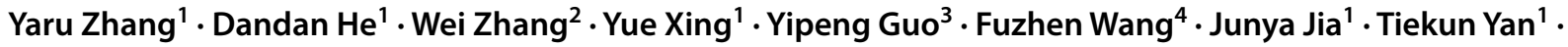 \\ Youxia Liu' ${ }^{1}$ Shan Lin ${ }^{1}$
}

Published online: 24 April 2020

(c) The Author(s) 2020

\begin{abstract}
Background The advantages of angiotensin-converting enzyme inhibitors (ACEIs) or angiotensin II receptor blockers (ARBs) in reducing risk of cardiovascular events (CVEs) and delaying end-stage kidney disease (ESKD) in patients with chronic kidney disease (CKD) is well-known. However, the efficacy and safety of these agents in non-dialysis CKD stages 3-5 patients are still a controversial issue.

Methods Two investigators (Yaru Zhang and Dandan He) independently searched and identified relevant studies from MEDLINE (from 1950 to October 2018), EMBASE (from 1970 to October 2018), and the Cochrane Library database. Randomised clinical trials in non-dialysis CKD3-5 patients treated with renin-angiotensin system (RAS) inhibitors were included. We used standard criteria (Cochrane risk of bias tool) to assess the inherent risk of bias of trials. We calculated the odds ratio (OR) and 95\% confidence interval (CI) for each outcome by random-effects model. A 2-sided $p$ value $<0.05$ was considered statistically significant, and all statistical analyses were performed using STATA, version 15.0. This network meta-analysis was undertaken by the frequency model.

Results Forty-four randomised clinical trials with 42,319 patients were included in our network meta-analysis. ACEIs monotherapy significantly decreased the odds of kidney events (OR $0.54,95 \%$ CI $0.41-0.73$ ), cardiovascular events (OR 0.73 , 95\% CI 0.64-0.84), cardiovascular death (OR 0.73, 95\% CI 0.63-0.86) and all-cause death (OR 0.77, 95\% CI 0.66-0.91) when compared to placebo. According to the cumulative ranking area (SUCRA), ACEI monotherapy had the highest probabilities of their protective effects on outcomes of kidney events (SUCRA 93.3\%), cardiovascular events (SUCRA 77.2\%), cardiovascular death (SUCRA 86\%), and all-cause death (SUCRA 94.1\%), even if there were no significant differences between ACEIs and other antihypertensive drugs, including calcium channel blockers (CCBs), $\beta$-blockers and diuretics on above outcomes except for kidney events. ARB monotherapy and combination therapy of an ACEI plus an ARB showed no more advantage than CCBs, $\beta$-blockers and diuretics in all primary outcomes. In the subgroup of non-dialysis diabetic kidney disease patients, no drugs, including ACEIs or ARBs, significantly lowered the odds of cardiovascular events and all-cause death. However, ACEIs were still better than other antihypertensive drugs including ARBs in all-cause death but not ARBs in cardiovascular events according to the SUCRA. Only ARBs had significant differences in preventing the occurrence of kidney events compared with placebo (OR 0.82, 95\% CI 0.72-0.95). Both ACEI/ARB monotherapy and combination therapy had higher odds of hyperkalaemia. ACEIs had 3.81 times higher odds than CCBs (95\% CI 1.58-9.20), ARBs had 2.08-5.10 times higher odds than placebo and CCBs and combination therapy of an ACEI and an ARB had 4.80-24.5 times higher odds than all other treatments. Compared with placebo, CCBs and $\beta$ blockers, ACEI therapy significantly increased
\end{abstract}

Yaru Zhang and Dandan He contributed equally to this study.

Electronic supplementary material The online version of this article (https://doi.org/10.1007/s40265-020-01290-3) contains supplementary material, which is available to authorized users.

Extended author information available on the last page of the article 
the odds of cough (OR 2.90, 95\% CI 1.76-4.77; OR 8.21, 95\% CI 3.13-21.54 and OR 1.80, 95\% CI 1.08-3.00). There were no statistical differences in hypotension among all comparisons except ACEIs versus placebo.

Conclusions Although ACEIs increased the odds of hyperkalaemia, cough and hypotension, they were still superior to ARBs and other antihypertensive drugs and had the highest benefits for the prevention of kidney events, cardiovascular outcomes, cardiovascular death and all-cause mortality in non-dialysis CKD3-5 patients. In patients with advanced diabetic kidney disease, ACEIs were superior to ARBs in lowering risk of all-cause death but not in kidney events and cardiovascular events.

\section{Key Points}

Chronic kidney disease (CKD) is a worldwide public health problem associated with a high prevalence of cardiovascular disease (CVD), impaired quality of life and all-cause mortality.

ACE inhibitors (ACEIs) and angiotensin II receptor blockers(ARBs) provide significant renal and cardiovascular protection for CKD patients, but the efficacy and safety of these agents in non-dialysis CKD3-5 patients is still uncertain.

This network meta-analysis demonstrates that ACEIs are superior to ARBs and other antihypertensive agents in reducing adverse cardiovascular and renal events, and all-cause mortality in non-dialysis CKD 3-5 patients, and are also superior to ARBs in lowering odds of allcause death but not in kidney events and cardiovascular events in the subgroup of patients with advanced CKD.

\section{Introduction}

Chronic kidney disease (CKD) is a global health burden with a high economic cost to health systems. The mean prevalence of CKD stages $1-5$ was $13.4 \%$ and stages $3-5$ was $10.6 \%$ [1]. CKD patients are at increased risks for cardiovascular disease (CVD) and all-cause mortality [2-5]. Lowering blood pressure (BP) is the foundation of management for slowing the progression of CKD [6], as well as a key point of management to reduce the risk for cardiovascular disease [7, 8]. Angiotensin-converting enzyme inhibitors (ACEIs) or angiotensin II receptor blockers (ARBs) are the best-studied antihypertensive agents that provide significant renal and cardiovascular protection for CKD patients [9], and have been recommended to be first-line therapy for patients with nondiabetic CKD, especially those with proteinuria by KDIGO (Kidney Disease: Improving Global Outcomes) clinical practice guideline [10]. The guideline also suggests that ACEIs or ARBs should be preferred therapies in patients with diabetic CKD and proteinuria, even in the absence of high blood pressure [10]. Unfortunately, most studies tested the renal or cardiovascular benefit of ACEIs or ARBs in the earlier stages of CKD, and few studies focused on patients with advanced CKD3-5. It still remains uncertain whether ACEIs or ARBs delay end-stage kidney disease (ESKD) and/or reduce mortality in these non-dialysis CKD3-5 patients. Network meta-analysis can compare multiple treatments by combining direct and indirect evidence to assess the interrelationships among all treatments. Many previous medical studies have proven its usefulness, which can unify and analyse the data from randomised clinical trials to compare multiple treatments without destroying the treatment in each trial [11-13]. We therefore undertook a systematic review and network meta-analysis to evaluate the effect of ACEIs or ARBs on kidney disease and cardiovascular outcomes in individuals with CKD3-5 but not on dialysis.

\section{Method}

\subsection{Data Sources and Searches}

Two investigators (Yaru Zhang and Dandan He) independently searched and identified relevant studies from the following data sources: MEDLINE (from 1950 to October 2018), EMBASE (from 1970 to October 2018), and the Cochrane Library database using PubMed and Ovid search engines with the text words of randomised controlled trial, chronic kidney disease, and all spellings of known ACE inhibitors and ARBs. Trials were considered without language restrictions. We also searched relevant reference lists from identified trials and review articles. When detailed information that was needed for the analysis was not available, we wrote to the author to request the data.

\subsection{Study Selection}

In this meta-analysis, we collected all randomised controlled trials (RCTs) regarding comparison of therapeutic effects of ACEIs/ARBs and other antihypertensive drugs in CKD3-5 participants who did not receive dialysis therapy. Inclusion criteria for studies were as follows: (1) study population comprised participants aged $\geq 18$ years with CKD3-5 who did not receive dialysis therapy [CKD3-5 was defined 
as glomerular filtration rate (GFR) $<60 \mathrm{~mL} / \mathrm{min} / 1.73 \mathrm{~m}^{2}$, elevated serum creatinine level]; (2) the intervention were ACEIs and/or ARBs and comparisons were placebo and/ or control therapy (defined as conventional therapy that specific drug type or name for control group was not provided in included studies) and/or other antihypertensive drugs; (3) reported study outcomes included kidney events (defined as a composite of any of the following: doubling of serum creatinine level, 50\% decline in GFR, or ESKD), and/or major cardiovascular events (defined as a composite of fatal or nonfatal myocardial infarction, stroke, and heart failure; cardiovascular death; or comparable definitions used by individual authors), and/or all-cause death, and/or drugrelated adverse events (including hyperkalaemia, cough, hypotension, and oedema); (4) study design was RCT, in which ACEIs or ARBs were given for at least 6 months, follow-up time was longer than 6 months and ample size was larger than 20 . We excluded studies (or sub-studies) aimed at CKD participants who have a functioning kidney transplant or who have chronic kidney disease treated with dialysis. We also excluded trials for CKD patients with GFR $>60 \mathrm{~mL} / \mathrm{min} / 1.73 \mathrm{~m}^{2}$ or normal serum creatinine level or trials without specified stage or trials lack of available data for CKD patients with GFR $<60 \mathrm{~mL} / \mathrm{min} / 1.73 \mathrm{~m}^{2}$.

\subsection{Data Extraction and Quality Assessment}

We extracted information using standard data extraction forms, which included baseline patient characteristics, intervention, dose of drug, follow-up duration, outcome events, and adverse events. These data were extracted from either studies which were conducted only for CKD3-5 patients or studies which included subgroup populations with CKD3-5 at baseline. We used standard criteria (Cochrane risk of bias tool) to assess the inherent risk of bias of trials, as showed in Table S1. Two investigators (Yaru Zhang and Dandan $\mathrm{He}$ ) independently undertook data extraction and quality assessment using a standardised approach. Any disagreements between the two investigators were resolved by consultation with a third reviewer (Youxia Liu).

\subsection{Data Synthesis and Statistical Analyses}

Pairwise meta-analysis and network meta-analysis were undertaken simultaneously. For both pairwise meta-analysis and network meta-analysis, we calculated the odds ratio (OR) and $95 \%$ confidence interval (CI) for each outcome by the randomeffects model. A 2-sided $p$ value $<0.05$ was considered statistically significant, and all statistical analyses were performed using STATA, version 15.0. This network meta-analysis was undertaken by the Frequentist model. For each outcome, we presented all treatment comparisons using a network graph. Loop-specific inconsistency approach was used to assess the disagreement between direct and indirect evidence in the loop, and the consistency results were considered not significant when $95 \%$ CIs of inconsistency factors included zero or RoR included one. RoR is defined as the difference that $\mathrm{OR}$ value of direct evidence minus OR value of indirect evidence. Treatment strategies were ranked for each outcome by the surface under the cumulative ranking curve (SUCRA) probabilities; and higher SUCRA probabilities in each simulation indicate the higher chance of being the best treatment regimen. We assessed publication bias in studies contributing to primary outcomes and adverse events by visual inspection of funnel plots performed using STATA, version 15.0.

\section{Results}

The literature search yielded original 7972 articles. During the process, studies such as VA NEPHRON-D [14] were eliminated owing to lack of available data for CKD subgroup populations with GFR $<60 \mathrm{~mL} / \mathrm{min} / 1.73 \mathrm{~m}^{2}$. Eventually, 44 RCTs with 42,139 patients were eligible for our network meta-analysis (Fig. 1). Of the included 44 RCTs, 33 studies provided kidney events, 30 provided cardiovascular events, 19 provided cardiovascular death, and 30 provided all-cause death. Thirty-three studies reported data on side effects, with 22 trials reporting hyperkalaemia, 18 reporting cough, 12 reporting oedema and nine reporting hypotension. The sample size ranged from 38 to 5662, and the follow-up for patients ranged from 1 to 5.3 years. The characteristics of the included studies are summarised in Table S2. Networks of eligible comparisons for the primary outcomes and side effects are showed in Figs. 2 and 3.

\subsection{Primary Outcomes}

Primary outcomes included kidney events, cardiovascular events, cardiovascular death, and all-cause death.

\subsubsection{Kidney Events}

Data regarding the effects of ACEIs or ARBs on kidney events were available from 33 trials with 23,766 patients. As showed in Figs. 4a, 5a, ACEI monotherapy was more effective than placebo (OR $0.54,95 \%$ CI $0.41-0.73$ ), control (OR 0.54, 95\% CI 0.33-0.87), calcium channel blockers (CCB) (OR 0.67, 95\% CI 0.50-0.89), $\beta$-blockers (OR $0.60,95 \%$ CI $0.37-0.96$ ) and ARBs (OR $0.71,95 \%$ CI $0.52-0.97)$. ARB monotherapy was only better than placebo (OR 0.76, 95\% CI 0.58-1.00). Combination therapy of an ACEI plus an ARB was not superior to ACEI or ARB monotherapy, control and other antihypertensive drugs. The effects of all drugs were ranked with SUCRA probabilities (Fig. 6a), and ACEI monotherapy had the greatest 
probability (SUCRA 93.3\%) for being the best treatment option on reducing renal events, followed by the combination of an ACEI plus an ARB (SUCRA 69.6\%) and diuretics (SUCRA 61.4\%), ARBs ranked in the fourth position (SUCRA 56.3\%) and placebo ranked last (SUCRA 17.5\%).

\subsubsection{Cardiovascular Events}

Thirty studies involving 34,538 patients evaluated the effect of ACEIs or ARBs on cardiovascular outcomes. As shown in Figs. 4b, 5b, compared to placebo, both ACEIs and ARBs reduced the odds of cardiovascular events (OR 0.73, 95\% CI $0.64-0.84$ and OR $0.83,95 \%$ CI $0.70-0.98$ ). ACEIs also have a significant benefit for cardiovascular protection (OR $0.66,95 \%$ CI $0.45-0.98$ ) compared to control group. However, neither ACEIs nor ARBs had any superiority of cardiovascular protection over diuretics, CCBs and $\beta$-blockers.
ACEI plus ARB therapy did not show a significant odds reduction for cardiovascular events compared to ACEI or ARB monotherapy (Figs. 4b, 5b). Although ACEIs were not significantly superior to diuretics, CCBs or $\beta$-blockers for lowering the odds of cardiovascular events according to forest map results, ACEIs still had the highest probability of being the best treatment option (Fig. 6b, SUCRA 77.2\%). ARBs (SUCRA 47.7\%) and ACEIs plus ARBs (SUCRA $47.1 \%$ ) ranked in the fifth and sixth position behind the CCBs (SUCRA 75.2\%), diuretics (SUCRA 66.2\%) and $\beta$-blockers (SUCRA 62.4\%).

\subsubsection{Cardiovascular Death}

Cardiovascular death events were reported from a total of 19 studies of 21,862 patients. ACEIs significantly decreased the rate of cardiovascular death compared with placebo
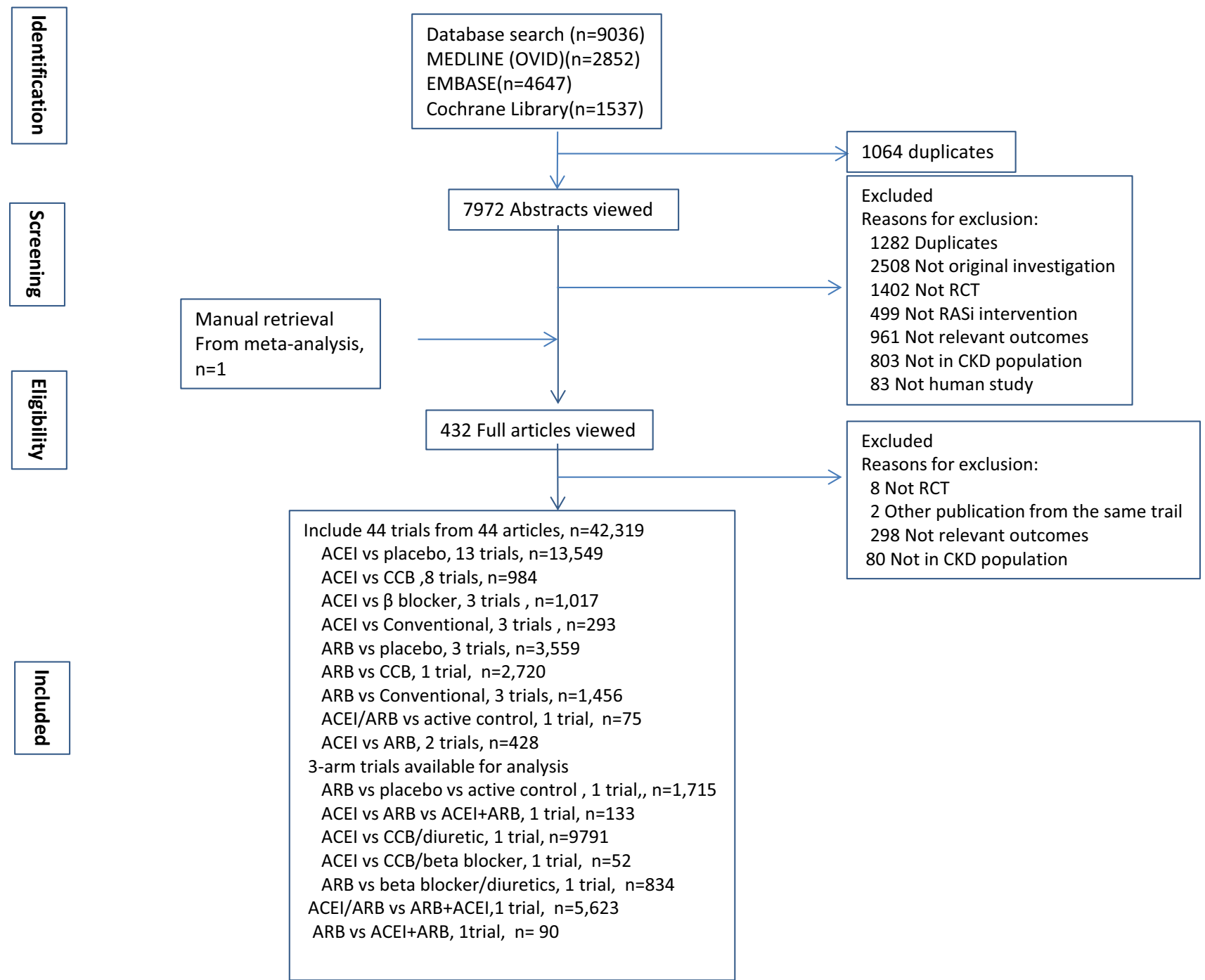

Fig. 1 Process for identifying studies eligible for the meta-analysis 


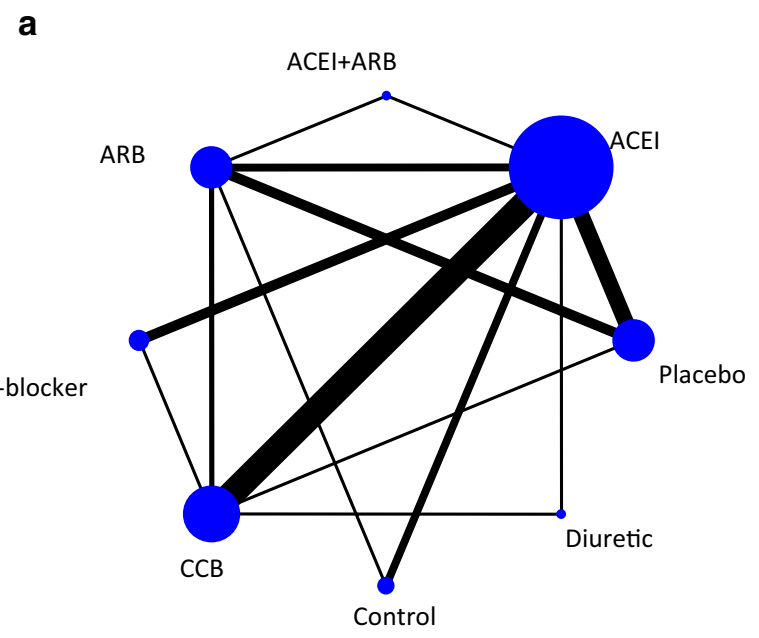

C

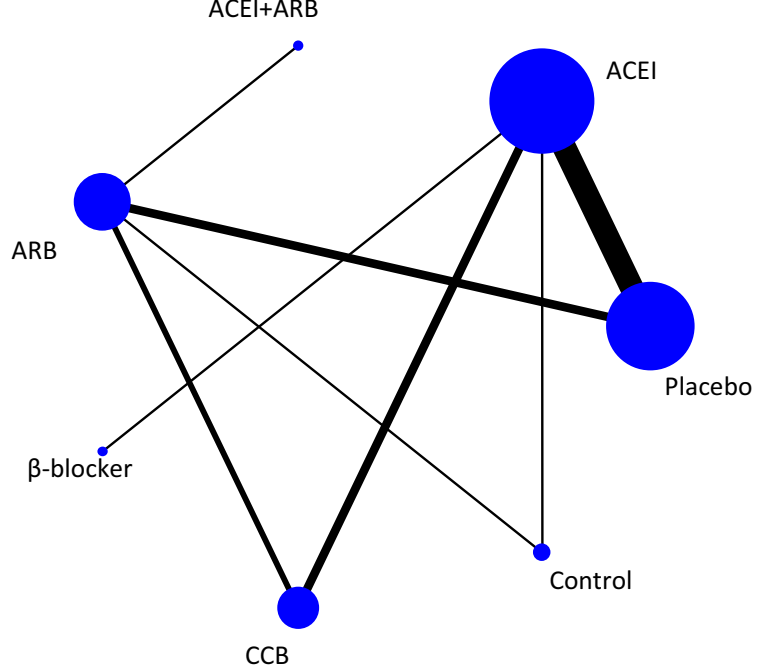

b

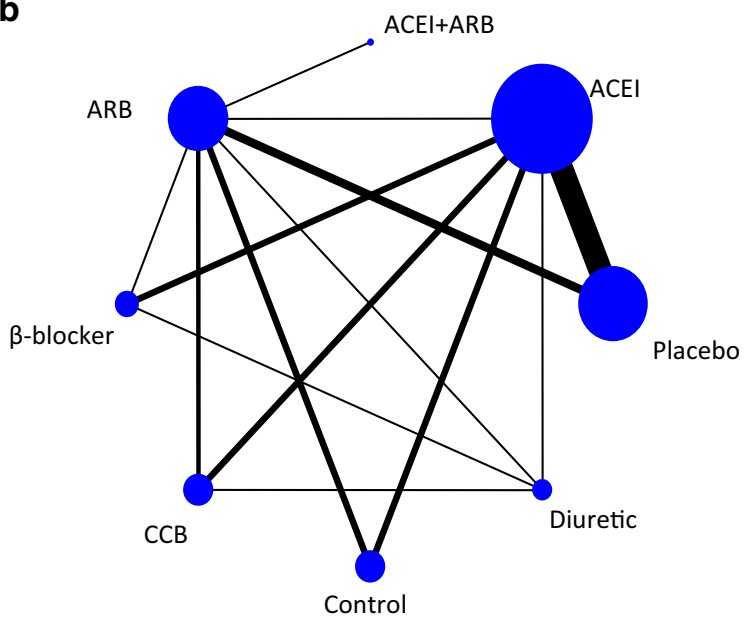

d

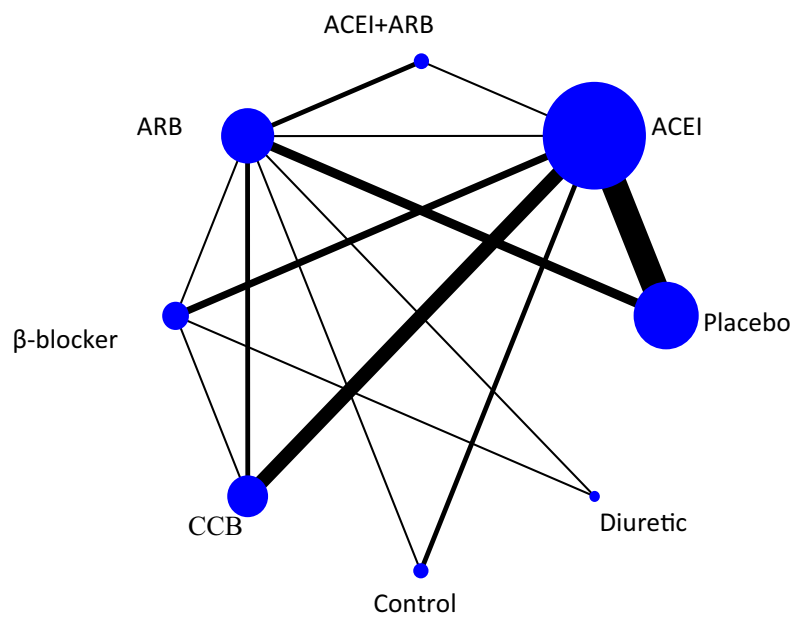

Fig. 2 Network of eligible treatment comparisons for a kidney events, $\mathbf{b}$ cardiovascular events, $\mathbf{c}$ cardiovascular death, and $\mathbf{d}$ all-cause death

(Figs. 4c, 5c, OR 0.73, 95\% CI 0.63-0.86), ARBs (Figs. 4c, $5 \mathrm{c}$, OR $0.63,95 \% \mathrm{CI} 0.46-0.86)$ and the combination therapy of ACEIs plus ARBs (Figs. 4c, 5c, OR 0.63, 95\% CI 0.42-0.94). Comparisons among other treatments, such as ARBs versus CCBs, did not show significant differences (Figs. 4c, 5c). The therapy of ACEIs in combination with ARBs was not superior to other treatments. ACEIs reduced the incidence of cardiovascular mortality at the top-ranking position (Fig. 6c, SUCRA $86.0 \%$ ) followed by $\beta$-blockers (SUCRA 74.9\%). Two of the least effective treatments for reducing cardiovascular death were control therapy (SUCRA 26.1\%) and ARBs (SUCRA 26.5\%).

\subsubsection{All-Cause Mortality}

Thirty studies with 26,144 participants evaluated the effect of ACEIs or ARBs in all-cause mortality. When studies were analyzed for ACEIs as the intervention, the likelihood of all-cause mortality was reduced by $23 \%, 40 \%, 24 \%$ compared with placebo, $\beta$-blockers and ARBs. ARB monotherapy or plus ACEI had no advantages in reducing all-cause mortality over other treatments (Figs. 4d, 5d). As can be seen in Fig. 6d, regarding prevention of all-cause mortality, ACEIs (SUCRA 94.1\%) were the most effective, followed by placebo (SUCRA 58.9\%), CCBs (SUCRA 58.8\%), ARBs (SUCRA 56.3\%), ACEIs plus ARBs (SUCRA 45.3\%). Diuretics were least effective (SUCRA25.5\%).

\subsection{Adverse Events}

Several major drug-related adverse outcomes including hyperkalaemia, cough, oedema and hypotension were analyzed. Both ACEIs and ARBs increased the odds of hyperkalaemia compared with CCBs (Fig. 7a, OR 3.81, 95\% CI 


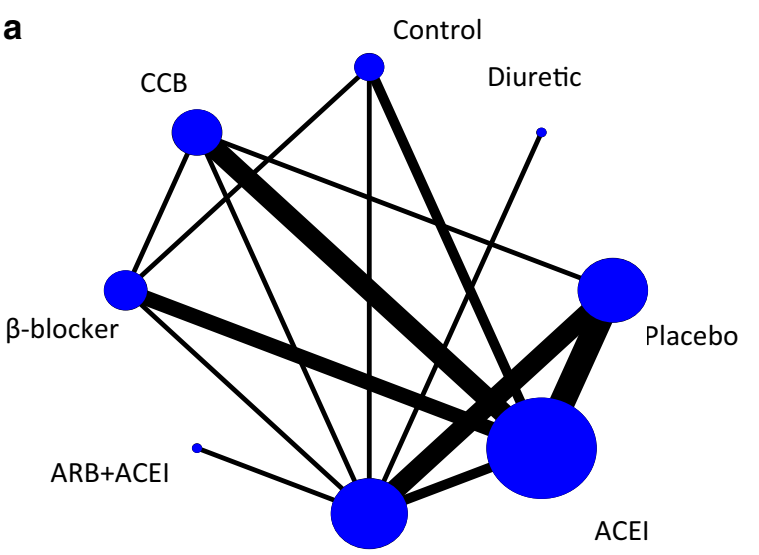

ARB

c

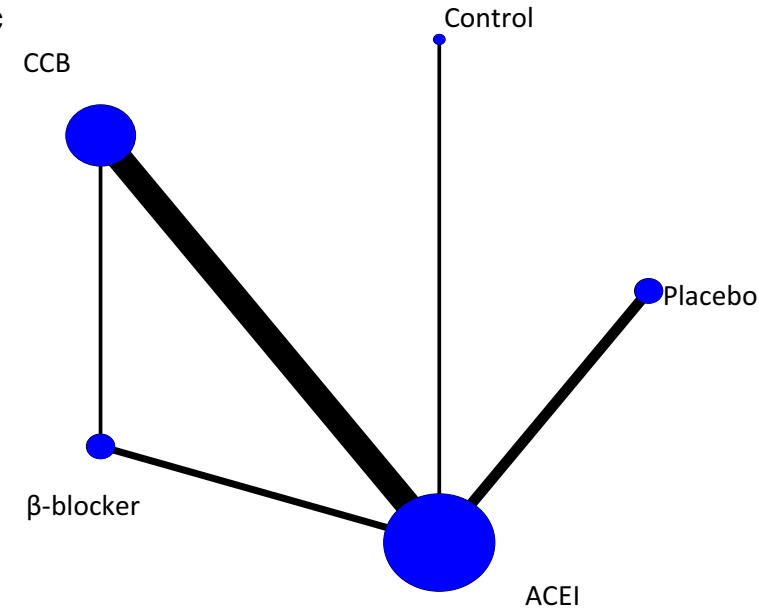

b

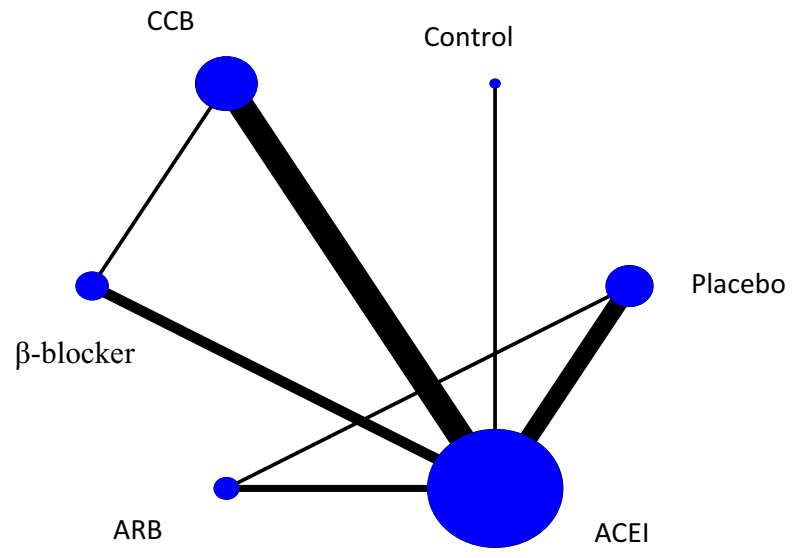

d

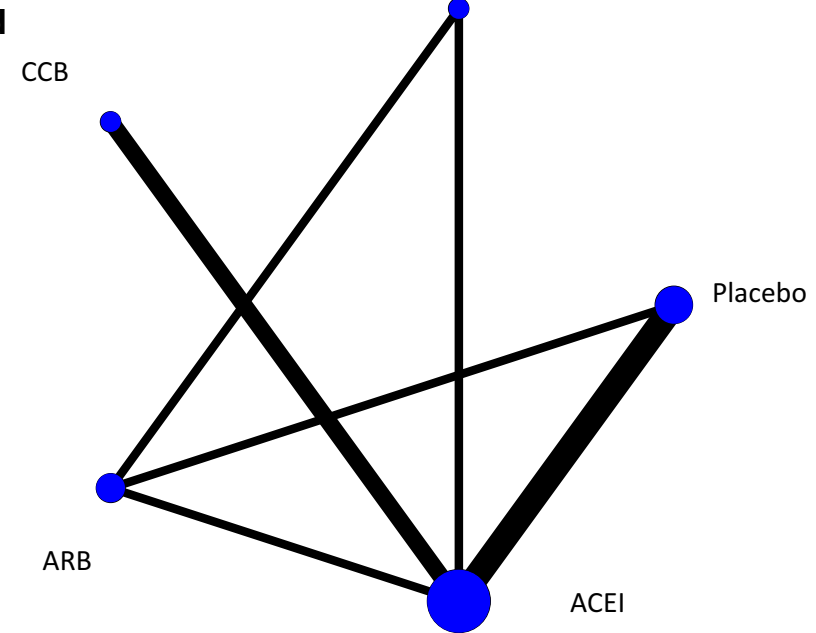

Fig. 3 Network of adverse events comparisons: a hyperkalaemia $\mathbf{b}$ cough $\mathbf{c}$ oedema $\mathbf{d}$ hypotension

1.58-9.20 and OR 5.10, 95\% CI 2.08-12.51). No statistically significant difference in the odds of hyperkalaemia was found between ACEI therapy and ARB therapy. The odds of hyperkalaemia in dual blockade therapy of RAS inhibitors is 4.80-24.5 times higher than that of all the other treatments included in our meta-analysis, including 6.43 times higher than ACEI monotherapy (95\% CI 3.79-10.92), 4.80 times higher than ARB monotherapy (95\% CI 4.04-5.70), and 24.5 times higher than CCBs (95\% CI 9.84-61.02). Compared with placebo, CCBs and $\beta$ blockers, ACEI therapy had statistically significant increases in the odds of cough (Fig. 7b, OR 2.90, 95\% CI 1.76-4.77; OR 8.21, 95\% CI 3.13-21.54 and OR 1.80, 95\% CI 1.08-3.00). The risk of edema with ACEIs was significantly lower than with CCBs (Fig. 7d, OR 0.61, 95\% CI 0.06-0.38), but had no statistical difference compared with placebo, control therapy, or $\beta$ blockers (Fig. 7d, OR 2.11, 95\% CI 0.33-13.53; OR 3.09, 95\% CI 0.11-87.94 and OR $0.65,95 \%$ CI $0.22-1.91)$. As for hypotension, there was no statistical difference among all comparisons except ACEIs versus placebo (Fig. 7c).

For increasing the incidence of hyperkalaemia, CCBs showed the highest safety ranking (Fig. 8, SUCRA 90.0\%), AECIs (SUCRA 33.6\%), ARBs (SUCRA 19.6\%), and ACEIs plus ARBs (SUCRA 0.0\%) showed the worst safety rankings. ACEIs (with the least SUCRA 6.8\% and SUCRA $10.1 \%$ ) had the highest possibility of cough and hypotension (Fig. 8). ARBs with higher safety for cough ranked second (SUCRA 71.4\%). ACEIs (SUCRA 56.5\%) ranked the first position for increasing the risk of oedema if taking no account of placebo (SUCRA 76.3\%) and control therapy (SUCRA 51.2\%).

\subsection{Loop-Specific Inconsistency Approach}

The results of direct and indirect estimates by the nodesplitting and loop-specific method were presented in Figs. S1 and S2. No significantly inconsistent loops were found 
a Treatment Effect Treatment Effect ACEI vs Placebo ACEI vs Control ACEI vs $C C B$ ACEI vs $\beta$-blocker ACEI vs $A C E I+A R B$ ACEI vs ARB ARB vs Placebo $A R B$ vs diuretic $A R B$ vs Control $A R B$ vs $C C B$ $A R B$ vs $\beta$-blocker $A R B$ vs $A C E I+A R B$ ACEI+ARB vs Placebo ACEI+ARBvs diuretic ACEI+ARB vs Control $A C E I+A R B$ vs $C C B$ ACEI+ARB vs $\beta$-blocker

3

C Treatment Effect

ACEI vs Placebo ACEI vs Control ACEI vs $C C B$ ACEI vs $\beta$-blocker ACEI vs ACEI+ARB ACEI vs ARB ARB vs Placebo ARB vs Control

$A R B$ vs $C C B$ $A R B$ vs $\beta$-blocker $A R B$ vs $A C E I+A R B$ ACEI+ARB vs Placebo $A C E I+A R B$ vs Control $A C E I+A R B$ vs $C$ CB ACEI+ARB vs $\beta$-blocker

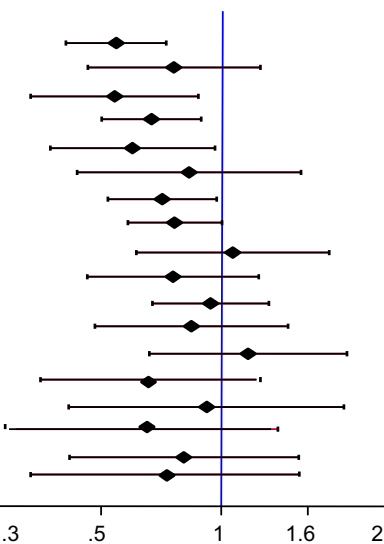

OR $(95 \% \mathrm{Cl})$ $0.54(0.41,0.73)$ $0.76(0.46,1.25)$ $0.54(0.33,0.87)$ $0.67(0.50,0.89)$ $0.60(0.37,0.96)$ $0.83(0.43,1.59)$ $0.71(0.52,0.97)$ $0.76(0.58,1.00)$ $1.07(0.61,1.87)$ $0.76(0.46,1.24)$ $0.94(0.67,1.32)$ $0.84(0.48,1.47)$ $1.17(0.66,2.07)$ $0.65(0.35,1.22)$ $0.92(0.41,2.04)$ $0.65(0.30,1.39)$ $0.80(0.41,1.56)$ $0.72(0.33,1.57)$

OR $(95 \% \mathrm{CI})$

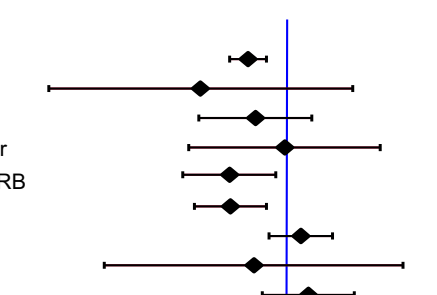

$0.73(0.63,0.86)$

$0.49(0.13,1.83)$

$0.78(0.48,1.28)$

$1.01(0.44,2.33)$

$0.63(0.42,0.94)$

$0.63(0.46,0.86)$

$1.16(0.88,1.53)$

$0.77(0.21,2.84)$

$1.24(0.83,1.86)$

$1.60(0.66,3.91)$

$0.99(0.77,1.28)$

$1.17(0.80,1.70)$

$0.78(0.21,2.92)$

$1.25(0.78,2.01)$

$1.61(0.64,4.07)$ b

Treatment Effect ACEI vs Placebo ACEI vs diuretic ACEI vs Contro
$A C E I$ v $C C B$ ACEI vs $\beta$-blocker $A C E I$ vs ACEI+ARB ACEI vs ARB ARB vs Placebo $A R B$ vs diuretic ARB vs Control ARB vs Control ARB vs CCB
ARB vs $\beta$-blocker ARB vs $\beta$-blocker
$A R B$ vs ACEI+ARB $A C E I+A R B$ vs Placebo ACEI+ARBvs diuretic $A C E I+A R B$ vs Control $A C E I+A R B$ vs $C C B$ ACEI+ARB vs $\beta$-blocker

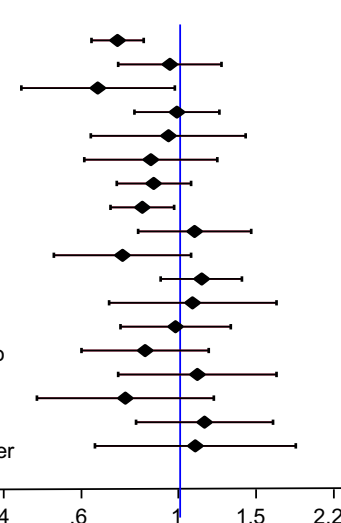

d Treatment Effect ACEI vs Placebo ACEI vs diuretic ACEI vs Control ACEI vs CCB ACEI vs $\beta$-blocker $A C E I$ vs $A C E I+A R B$ $A C E I$ vs $A R B$ ARB vs Placebo $A R B$ vs diuretic ARB vs Control $A R B$ vs CCB ARB vs $\beta$-blocker ARB VS ACEI+ARB ACEI+ARBvs diuretic $A C E I+A R B$ vs Control $A C E I+A R B$ vs $C C B$ ACEI+ARB vs $\beta$-blocker

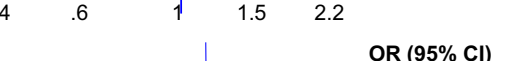
ACE
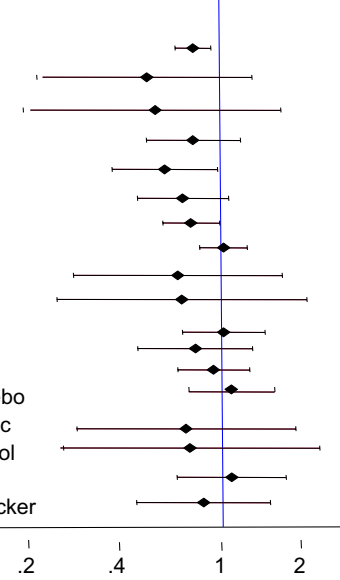

$0.77(0.66,0.91)$ $0.52(0.21,1.30)$ $0.54(0.18,1.62)$ $0.78(0.51,1.17)$ $0.60(0.38,0.96)$ $0.71(0.48,1.06)$ $0.76(0.59,0.98)$ $1.01(0.82,1.25)$ $0.68(0.27,1.70)$ $0.70(0.23,2.11)$ $1.02(0.71,1.46)$ $0.79(0.48,1.31)$ $0.93(0.68,1.28)$
$1.09(0.75,1.59)$ $0.73(0.28,1.92)$ $0.76(0.24,2.37)$ $1.09(0.68,1.77)$ $0.85(0.47,1.54)$

5.5

Fig. 4 Forest plot for results of primary outcomes: a kidney events, $\mathbf{b}$ cardiovascular events, $\mathbf{c}$ cardiovascular death, and $\mathbf{d}$ all-cause death

in the treatment networks of most events except those of cough with larger RoR value of the loop placebo-ARBsACEIs (RoR 95.285). Data were double checked, and we were unable to identify any significant effect modifier that differed across comparisons.

\subsection{Risks of Bias}

The results of publication bias in studies contributing to primary outcomes and adverse events were displayed in Figs. S4 and S5, and risks of bias were generally low.

\subsection{Subgroup Analyses and Quality Assessment}

The results of subgroup analyses for the primary outcomes and hyperkalaemia of each treatment versus placebo were presented in Table S3a-d and Table S4. In a subgroup of non-dialysis diabetic kidney disease patients, of the treatments listed in Table S3a, only ARBs had significant differences in preventing the occurrence of kidney events compared with placebo (Table S3a, OR 0.82, 95\% CI
0.72-0.95) and ARBs had superiority over ACEIs, CCBs, $\beta$-blockers and placebo ranking in the top position with highest SUCRA probability followed by ACEIs. No drugs, including ACEIs or ARBs, significantly lowered the odds of cardiovascular events and all-cause death, but ACEIs/ ARBs were still relatively superior to other drugs. Among ACEIs, ARBs, CCBs, and $\beta$-blockers, only ARBs significantly increased the odds of hyperkalaemia (Table S4, OR 2.15, 95\% CI 1.25-3.69) compared to placebo. ARBs ranked ahead of ACEIs in reducing the risk of cardiovascular events, but behind ACEIs for cardiovascular death, all-cause death and hyperkalaemia. ACEIs had the highest probability of being the best option in preventing all-cause death in this population. When we conducted subgroup analyses by inclusion of trials with serum creatinine $>200 \mu \mathrm{mol} / \mathrm{L}$, we found ACEIs were superior to ARBs in kidney events (Table S3a) but inferior to ARBs in cardiovascular events (Table S3b). The effects of ARBs for cardiovascular death and all-cause death were not analyzed due to few available data (Table S3c-d). Most results of all treatments for primary outcomes in patients with creatinine $<200 \mu \mathrm{mol} / \mathrm{L}$ were consistent with those of all 
Fig. 5 Summary of results from network meta-analysis (on the upper triangle) and traditional pairwise meta-analysis (on the lower triangle) on primary outcomes: a kidney events, b cardiovascular events, $\mathbf{c}$ cardiovascular death, and d all-cause death. On the lower triangle and upper triangle, the row-defining treatment is compared with the column-defining treatment, and ORs of $<1$ favour the rowdefining treatment. Significant results are shown in boldface type a Kidney events

\begin{tabular}{|c|c|c|c|c|c|c|c|}
\hline Placebo & $\begin{array}{c}0.71 \\
(0.41,1.24)\end{array}$ & $\begin{array}{c}1.01 \\
(0.60,1.70)\end{array}$ & $\begin{array}{c}0.81 \\
(0.58,1.14)\end{array}$ & $\begin{array}{c}0.91 \\
(0.53,1.57)\end{array}$ & $\begin{array}{c}0.65 \\
(0.35,1.22)\end{array}$ & $\begin{array}{c}0.76 \\
(0.58,1.00)\end{array}$ & $\begin{array}{c}0.54 \\
(0.41,0.73)\end{array}$ \\
\hline - & diuretic & $\begin{array}{c}1.41 \\
(0.71,2.81)\end{array}$ & $\begin{array}{c}1.14 \\
(0.69,1.88)\end{array}$ & $\begin{array}{c}1.27 \\
(0.64,2.53)\end{array}$ & $\begin{array}{c}0.92 \\
(0.41,2.04)\end{array}$ & $\begin{array}{c}1.07 \\
(0.61,1.87)\end{array}$ & $\begin{array}{c}0.76 \\
(0.46,1.25)\end{array}$ \\
\hline - & - & Control & $\begin{array}{c}0.81 \\
(0.47,1.38)\end{array}$ & $\begin{array}{c}0.90 \\
(0.46,1.78)\end{array}$ & $\begin{array}{c}0.65 \\
(0.30,1.39)\end{array}$ & $\begin{array}{c}0.76 \\
(0.46,1.24)\end{array}$ & $\begin{array}{c}0.54 \\
(0.33,0.87)\end{array}$ \\
\hline- & - & - & CCB & $\begin{array}{c}1.12 \\
(0.65,1.94)\end{array}$ & $\begin{array}{c}0.80 \\
(0.41,1.56)\end{array}$ & $\begin{array}{c}0.94 \\
(0.67,1.32)\end{array}$ & $\begin{array}{c}0.67 \\
(0.50,0.89)\end{array}$ \\
\hline- & - & 一 & - & $\beta$-blocker & $\begin{array}{c}0.72 \\
(0.33,1.57)\end{array}$ & $\begin{array}{c}0.84 \\
(0.48,1.47)\end{array}$ & $\begin{array}{c}0.60 \\
(0.37,0.96)\end{array}$ \\
\hline 一 & - & 一 & - & 一 & ACEI+ARB & $\begin{array}{c}1.17 \\
(0.66,2.07)\end{array}$ & $\begin{array}{c}0.83 \\
(0.43,1.59)\end{array}$ \\
\hline $\begin{array}{c}0.89 \\
(0.82,0.97)\end{array}$ & - & $\begin{array}{c}0.74 \\
(0.54,1.01)\end{array}$ & - & - & - & ARB & $\begin{array}{c}0.71 \\
(0.52,0.97)\end{array}$ \\
\hline $\begin{array}{c}0.60 \\
(0.49,0.72)\end{array}$ & - & $\begin{array}{c}0.86 \\
(0.57,1.28)\end{array}$ & $\begin{array}{c}0.95 \\
(0.80,1.13)\end{array}$ & $\begin{array}{c}0.79 \\
(0.66,0.95)\end{array}$ & - & $\begin{array}{c}0.71 \\
(0.52,0.96)\end{array}$ & ACEI \\
\hline
\end{tabular}

b Cardiovascular events

\begin{tabular}{|c|c|c|c|c|c|c|c|}
\hline Placebo & $\begin{array}{c}0.76 \\
(0.58,1.01)\end{array}$ & $\begin{array}{c}1.11 \\
(0.75,1.63)\end{array}$ & $\begin{array}{c}0.74 \\
(0.59,0.92)\end{array}$ & $\begin{array}{c}0.77 \\
(0.51,1.17)\end{array}$ & $\begin{array}{c}0.84 \\
(0.61,1.17)\end{array}$ & $\begin{array}{c}0.83 \\
(0.70,0.98)\end{array}$ & $\begin{array}{c}0.73 \\
(0.64,0.84)\end{array}$ \\
\hline- & diuretic & $\begin{array}{c}1.45 \\
(0.92,2.28)\end{array}$ & $\begin{array}{c}0.96 \\
(0.74,1.26)\end{array}$ & $\begin{array}{c}1.01 \\
(0.64,1.61)\end{array}$ & $\begin{array}{c}1.10 \\
(0.73,1.66)\end{array}$ & $\begin{array}{c}1.09 \\
(0.81,1.46)\end{array}$ & $\begin{array}{c}0.96 \\
(0.73,1.25)\end{array}$ \\
\hline - & - & Control & $\begin{array}{c}0.67 \\
0.44,1.00)\end{array}$ & $\begin{array}{c}0.70 \\
0.40,1.21)\end{array}$ & $\begin{array}{c}0.76 \\
(0.48,1.20)\end{array}$ & $\begin{array}{c}0.75 \\
(0.53,1.07)\end{array}$ & $\begin{array}{c}0.66 \\
(0.45,0.98)\end{array}$ \\
\hline- & - & - & CCB & $\begin{array}{c}1.05 \\
(0.67,1.63)\end{array}$ & $\begin{array}{c}1.14 \\
(0.80,1.63)\end{array}$ & $\begin{array}{c}1.13 \\
(0.91,1.39)\end{array}$ & $\begin{array}{c}0.99 \\
(0.80,1.23)\end{array}$ \\
\hline - & - & - & - & $\beta$-blocker & $\begin{array}{c}1.09 \\
(0.65,1.83)\end{array}$ & $\begin{array}{c}1.08 \\
(0.70,1.66)\end{array}$ & $\begin{array}{c}0.95 \\
(0.64,1.42)\end{array}$ \\
\hline - & - & 一 & - & 一 & ACEI+ACEI & $\begin{array}{c}0.99 \\
(0.74,1.31)\end{array}$ & $\begin{array}{c}0.87 \\
(0.62,1.22)\end{array}$ \\
\hline $\begin{array}{c}0.92 \\
(0.84,1.01)\end{array}$ & $\begin{array}{c}1.18 \\
(0.45,3.13)\end{array}$ & $\begin{array}{c}0.82 \\
(0.68,0.99)\end{array}$ & $\begin{array}{c}1.01 \\
(0.87,1.78)\end{array}$ & $\begin{array}{c}1.11 \\
(0.43,2.83)\end{array}$ & $\begin{array}{c}0.99 \\
(0.89,1.10)\end{array}$ & ARB & $\begin{array}{c}0.88 \\
(0.73,1.07)\end{array}$ \\
\hline $\begin{array}{c}0.79 \\
.74,0.84)\end{array}$ & $\begin{array}{c}1.07 \\
(0.98,1.67)\end{array}$ & $\begin{array}{c}0.68 \\
(0.19,2.40)\end{array}$ & $\begin{array}{c}1.01 \\
(0.92,1.11)\end{array}$ & $\begin{array}{c}0.95 \\
0.69,1.31)\end{array}$ & - & $\begin{array}{c}1.00 \\
0.41,2.46)\end{array}$ & ACEI \\
\hline
\end{tabular}

C Cardiovascular death

\begin{tabular}{|c|c|c|c|c|c|c|}
\hline Placebo & $\begin{array}{c}1.50 \\
(0.40,5.62)\end{array}$ & $\begin{array}{c}0.94 \\
(0.58,1.51)\end{array}$ & $\begin{array}{c}0.73 \\
(0.31,1.69)\end{array}$ & $\begin{array}{c}1.17 \\
(0.80,1.70)\end{array}$ & $\begin{array}{c}1.16 \\
(0.88,1.53)\end{array}$ & $\begin{array}{c}0.73 \\
(0.63,0.86)\end{array}$ \\
\hline- & Control & $\begin{array}{c}0.62 \\
(0.16,2.42)\end{array}$ & $\begin{array}{c}0.48 \\
(0.10,2.30)\end{array}$ & $\begin{array}{c}0.78 \\
(0.21,2.93)\end{array}$ & $\begin{array}{c}0.77 \\
(0.21,2.84)\end{array}$ & $\begin{array}{c}0.49 \\
(0.13,1.83)\end{array}$ \\
\hline- & - & $\mathrm{CCB}$ & $\begin{array}{c}0.78 \\
(0.29,2.04)\end{array}$ & $\begin{array}{c}1.25 \\
(0.78,2.01)\end{array}$ & $\begin{array}{c}1.24 \\
(0.83,1.86)\end{array}$ & $\begin{array}{c}0.78 \\
(0.48,1.28)\end{array}$ \\
\hline- & - & - & $\beta$-blocker & $\begin{array}{c}1.61 \\
(0.64,4.07)\end{array}$ & $\begin{array}{c}1.60 \\
(0.66,3.91)\end{array}$ & $\begin{array}{c}1.014 \\
(0.44,33)\end{array}$ \\
\hline- & - & - & - & ACEI+ARB & $\begin{array}{c}0.99 \\
(0.77,1.28)\end{array}$ & $\begin{array}{c}0.63 \\
(0.42,0.94)\end{array}$ \\
\hline 0.91 & - & 1.01 & - & 1.01 & ARB & 0.63 \\
$(0.81,1.03)$ & - & $(0.87,1,18)$ & - & $(0.86,1.17)$ & & $(0.46,0.86)$ \\
\hline 0.80 & 0.33 & 1.50 & 0.95 & & - & ACEI \\
\hline$(0.75,0.86)$ & $(0.04,3.05)$ & $(0.28,8.18)$ & $(0.69,1.31)$ & - & & \\
\hline
\end{tabular}

d All-cause death

\begin{tabular}{|c|c|c|c|c|c|c|c|}
\hline Placebo & $\begin{array}{c}1.49 \\
(0.60,3.75)\end{array}$ & $\begin{array}{c}1.44 \\
(0.48,4.34)\end{array}$ & $\begin{array}{c}1.00 \\
(0.67,1.49)\end{array}$ & $\begin{array}{c}1.28 \\
(0.79,2.07)\end{array}$ & $\begin{array}{c}1.09 \\
(0.75,1.59)\end{array}$ & $\begin{array}{c}1.01 \\
(0.82,1.25)\end{array}$ & $\begin{array}{c}0.77 \\
(0.66,0.91)\end{array}$ \\
\hline - & diuretic & $\begin{array}{c}0.96 \\
(0.23,4.00)\end{array}$ & $\begin{array}{c}0.67 \\
(0.25,1.77)\end{array}$ & $\begin{array}{c}0.86 \\
(0.35,2.12)\end{array}$ & $\begin{array}{c}0.73 \\
(0.28,1.92)\end{array}$ & $\begin{array}{c}0.68 \\
(0.27,1.70)\end{array}$ & $\begin{array}{c}0.52 \\
(0.21,1.30)\end{array}$ \\
\hline- & - & control & $\begin{array}{c}0.69 \\
(0.22,2.19)\end{array}$ & $\begin{array}{c}0.89 \\
(0.27,2.92)\end{array}$ & $\begin{array}{c}0.76 \\
(0.24,2.37)\end{array}$ & $\begin{array}{c}0.70 \\
(0.23,2.11)\end{array}$ & $\begin{array}{c}0.54 \\
(0.18,1.62)\end{array}$ \\
\hline- & - & - & $\mathrm{CCB}$ & $\begin{array}{c}1.28 \\
(0.71,2.33)\end{array}$ & $\begin{array}{c}1.09 \\
(0.68,1.77)\end{array}$ & $\begin{array}{c}1.02 \\
(0.71,1.46)\end{array}$ & $\begin{array}{c}0.78 \\
(0.51,1.17)\end{array}$ \\
\hline \multirow[t]{2}{*}{-} & - & - & - & $\beta$-blocker & $\begin{array}{c}0.85 \\
(0.47,1.54)\end{array}$ & $\begin{array}{c}0.79 \\
(0.48,1.31)\end{array}$ & $\begin{array}{c}0.60 \\
(0.38,0.96)\end{array}$ \\
\hline & - & - & - & - & $A R B+A C E I$ & $\begin{array}{c}0.93 \\
(0.68,1.28)\end{array}$ & $\begin{array}{c}0.71 \\
(0.48,1.06)\end{array}$ \\
\hline $\begin{array}{c}1.03 \\
(0.91,1.17)\end{array}$ & & $\begin{array}{c}1.04 \\
(0.27,4.01)\end{array}$ & $\begin{array}{c}0.99 \\
(0.76,1.29)\end{array}$ & - & $\begin{array}{c}1.04 \\
(0.93,1.17)\end{array}$ & ARB & $\begin{array}{c}0.76 \\
(0.59,0.98)\end{array}$ \\
\hline $\begin{array}{c}0.84 \\
(0.76,0.92)\end{array}$ & & $\begin{array}{c}0.34 \\
(0.07,1.65)\end{array}$ & $\begin{array}{c}0.84 \\
(0.33,2.11)\end{array}$ & $\begin{array}{c}0.68 \\
(0.45,1.01)\end{array}$ & - & $\begin{array}{c}1.60 \\
(0.15,16.75)\end{array}$ & ACEI \\
\hline
\end{tabular}



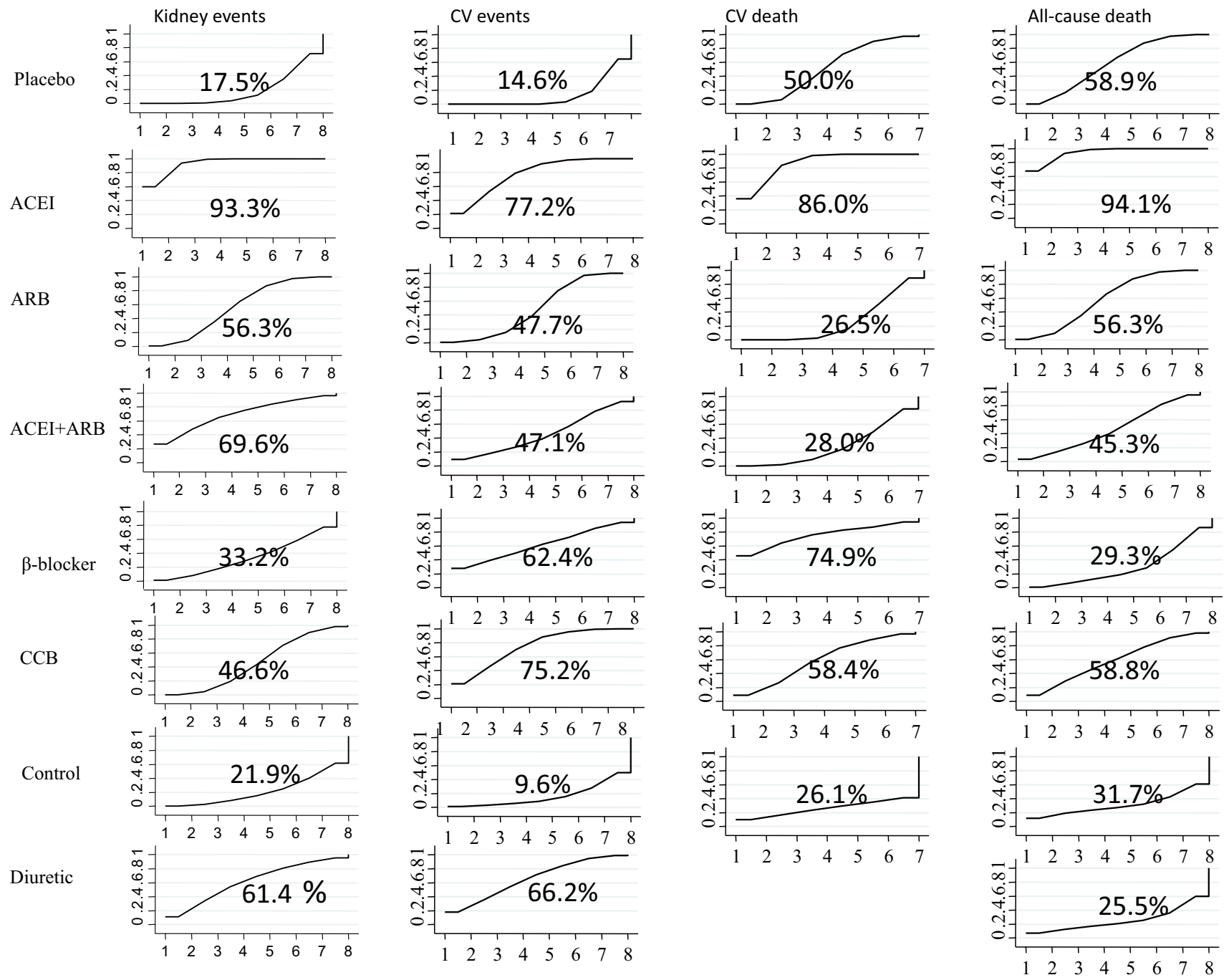

Fig. 6 Ranking of treatment strategies based on probability of their protective effects on outcomes of kidney events, cardiovascular events, cardiovascular death, and all-cause death according to the cumulative ranking area (SUCRA). Larger probability, stronger protective effects

patients with non-dialysis CKD3-5. The odds of hyperkalaemia with ARBs was 2.09 times higher than placebo in patients with creatinine $>200 \mu \mathrm{mol} / \mathrm{L}$, but ACEIs did not produce significant discrepancies whether creatinine was $>200 \mu \mathrm{mol} / \mathrm{L}$ or $<200 \mu \mathrm{mol} / \mathrm{L}$. When sample size was larger than 100 and follow-up longer than three years, the likelihood of all primary outcomes in ACEI users was still significantly lower than those in placebo group, and ACEIs still had the greatest probability of being the best treatment option for CKD3-5 patients (Table S3a-d). Most results of curative effect sequencing were generally consistent with those in standard analysis. Notably, in studies with sample size larger than 100, ACEIs and $\beta$-blockers involved significantly increased risk of hyperkalaemia compared to placebo, which was something different (Table S4).
The inherent risk of bias of trials was performed for all studies, and only 11 had low risk of bias of each item in the Cochrane risk-of-bias tool (including sequence generation, allocation concealment, blinding, incomplete outcome data, selective outcome reporting, and other sources of bias).

After excluding all studies where any item of the Cochrane risk of bias assessments was high or unclear, a subgroup analysis of 11 studies showed that ACEIs had significant differences in preventing the occurrence of all primary outcomes compared with placebo. ARBs had superiority over placebo only in preventing cardiovascular events. ACEIs had significant advantages over ARBs in preventing all primary outcomes (Table S5 and Figure S6), which was generally consistent with the forest plot results of all studies included in this meta-analysis (Fig. 4). 


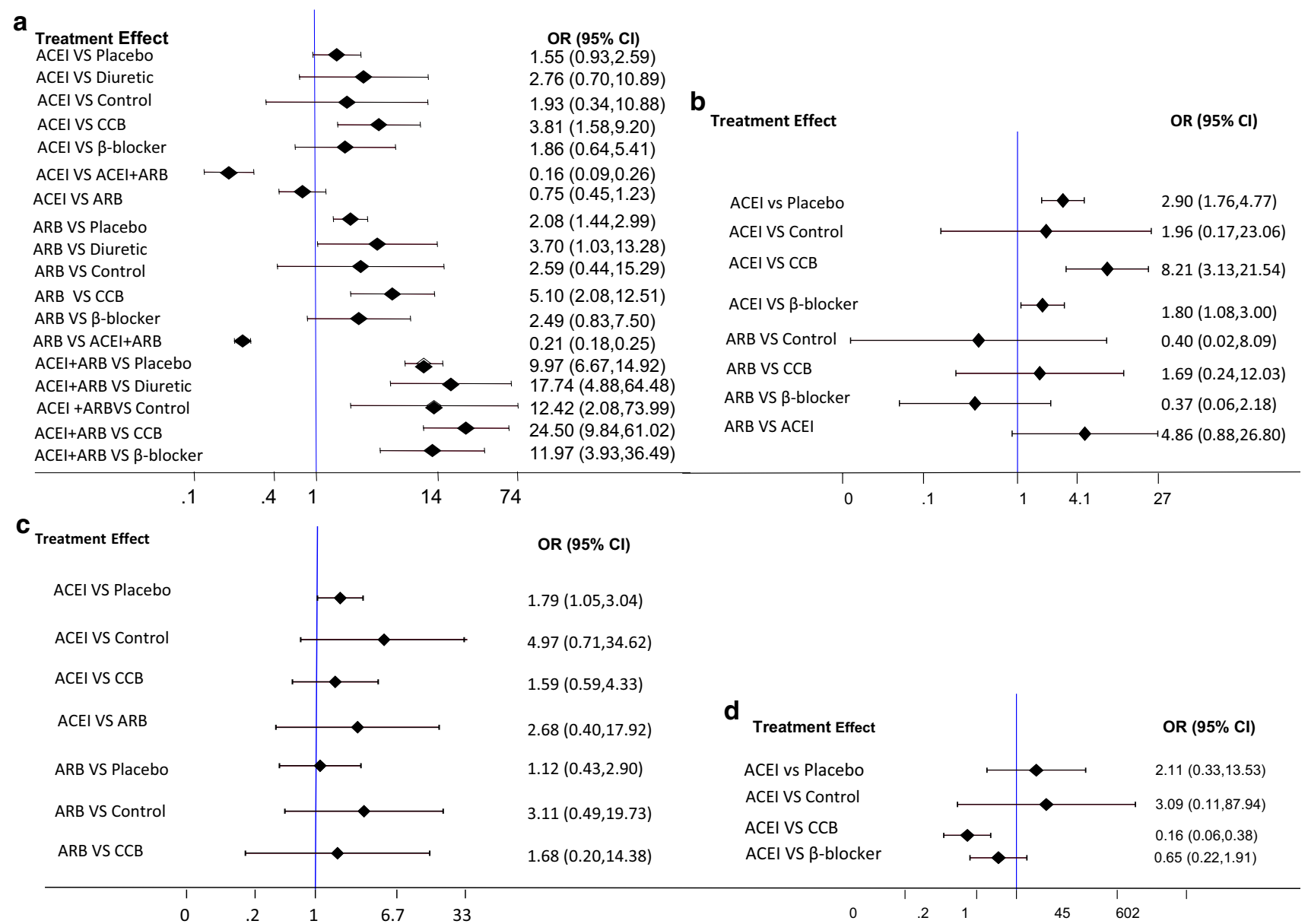

Fig. 7 Forest plot for results of adverse events comparisons: a hyperkalaemia $\mathbf{b}$ cough $\mathbf{c}$ hypotension $\mathbf{d}$ oedema

\section{Discussion}

This network meta-analysis, pooling evidence from RCTs that each trial may compare different treatments, was conducted simultaneously to estimate relative effectiveness and safety of renin-angiotensin-aldosterone system (RAAS)blocking agents for any pair of treatments on kidney disease and cardiovascular outcomes in individuals with CKD3-5 but not on dialysis. Thus, overcoming the lack of comparative data in head-to-head trials. Eventually, 44 RCTs with 42,139 patients met our inclusion criteria. Our results suggested that although RAS blockade therapy definitely increased the likelihood for hyperkalaemia, hypotension, and cough in patients with CKD3-5, it was still more beneficial to protect renal and cardiovascular functions. ACEIs, superior to ARBs and other antihypertensive drugs, had the highest probability of being the most beneficial treatments for kidney events, cardiovascular outcomes and all-cause mortality in non-dialysis CKD3-5 patients. In patients with diabetic kidney disease, ARBs were superior to ACEIs in lowering odds of kidney and cardiovascular events, but fell behind ACEIs in risk of all-cause death.

Several published meta-analyses and systematic reviews evaluated the effects of RAS blockers on CKD patients [9, 15-21]. One network meta-analysis that included 119 RCTs comprising 64,768 participants, mostly with chronic kidney disease, showed that ACEIs or ARBs could reduce the risks of kidney failure and cardiovascular events. However, these studies targeting CKD patients, making the effect of RAS blockades unclear for patients with CKD3-5. Our findings suggested that ACEI monotherapy was significantly better than placebo, control therapy, CCBs, $\beta$-blockers and ARBs in reducing the odds of kidney failure, and was also superior to placebo, $\beta$-blockers and ARBs in reducing the odds of all-cause mortality in patients with non-dialysis CKD3-5. Although ACEI monotherapy made no significant differences in cardiovascular events and cardiovascular death compared to the therapy of other antihypertensive agents, it was still most likely to be the best treatment option in reducing the odds of cardiovascular events, cardiovascular 

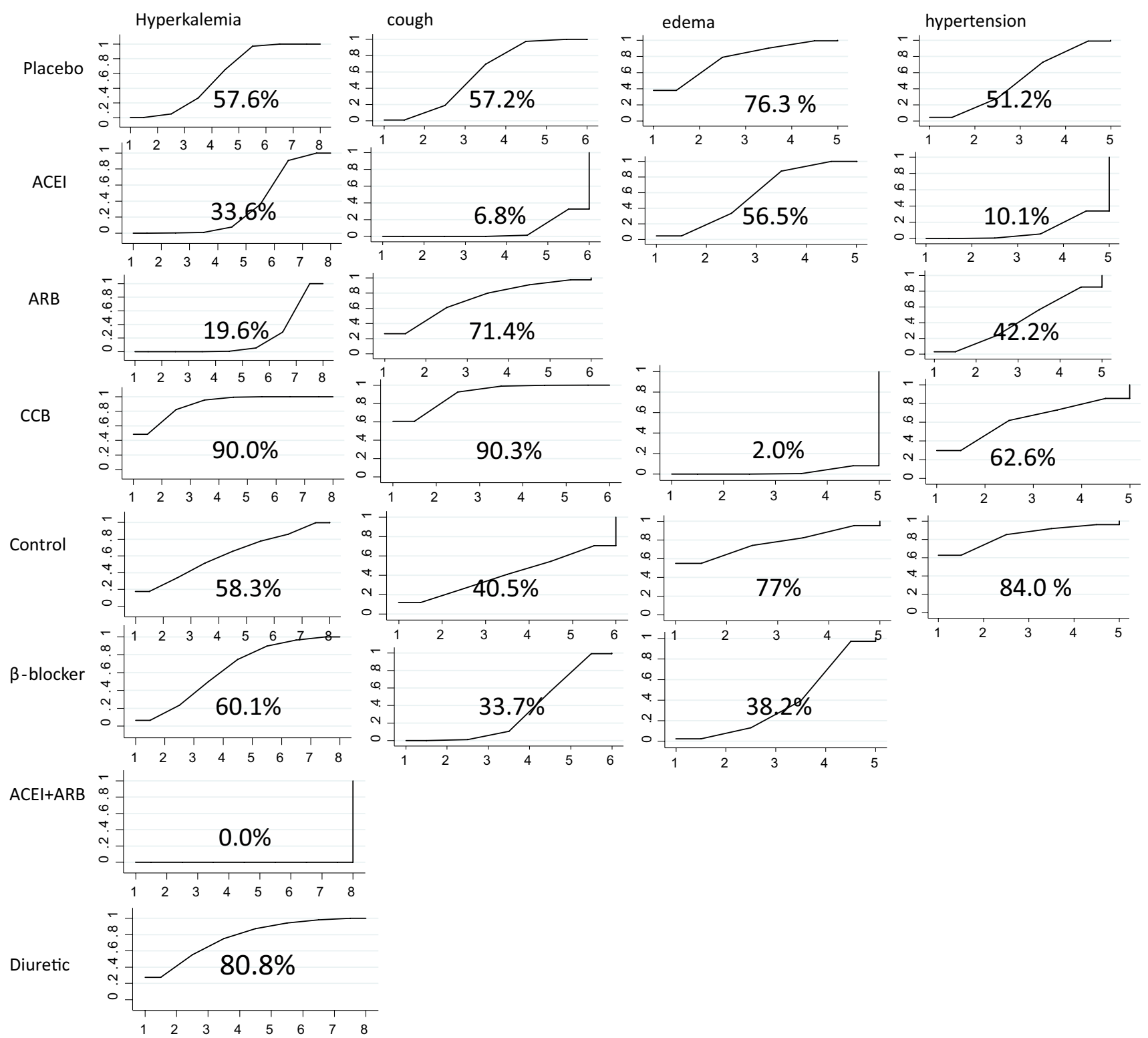

Fig. 8 Ranking of treatment strategies based on probability of their protective effects on outcomes of for hyperkalaemia, cough, oedema and hypotension according to the cumulative ranking area (SUCRA). Larger probability, stronger protective effects

death in individuals with non-dialysis CKD3-5 according to SUCRA probabilities. Besides, ARBs were only superior to placebo for kidney and cardiovascular events, and had no advantages over all other antihypertensive drugs for cardiovascular death and all-cause death. Consistent with our findings, available data showed the benefits of ACEIs were stronger than those of ARBs. A meta-analysis in which 119 RCTs $(n=64,768)$ were included suggested that ACEIs were more effective than ARBs in protecting against kidney failure, cardiovascular death, and all-cause death in CKD patients [9]. A recent network meta-analysis of 63 trials (36,917 patients) also found that ACEIs produced more benefits to CKD patients in mortality and kidney events [17]. The present study provided additional evidence to support the viewpoint that ACEIs protect the kidney and reduce the risk of cardiovascular disease and death in CKD3-5 patients, potentially to a greater degree than that achieved with ARBs. This disparity may be contributed to by different mechanisms of action of ACEIs and ARBs on the RAS pathway [9]. ACEI inhibits the conversion of Ang I to Ang II, while ARB selectively prevents Ang II from binding to AT1R. Both drugs can prevent the harmful effects of Ang II overproduction on the cardiovascular system. However, ACEI is superior to ARB in pharmacological action mechanism. 
Studies have shown that ACEI increases Ang (one of the important biologically active substances in RAAS) (1-7) levels 10- to 25-fold [22]. As a substrate for ACE, Ang (1-7) competes with Ang I and bradykinin (BK) for degradation, thereby inhibiting the formation of Ang II and enhancing BK activity. ARB usually preferentially blocks AT1R and stimulates angiotensin 2 (AT2R) and angiotensin IV receptor (AT4R) [23-27]. Compared with AT1R, the signalling pathways promoted by AT2R are far from being resolved. Studies have found that AT2R stimulation produces an autophagy vasodilator cascade consisting of BK, nitric oxide (NO) and guanosine ring 3',5'monophosphate (cGMP) [28, 29]. Therefore, AT2R activation may have the opposite effect to those of AT1R activation [30, 31]. Therefore, stimulating AT2R during the blocking of AT1 with ARB will produce a beneficial response. Unfortunately, data suggest that AT2R stimulation may not be as beneficial as previously suggested, and may even be harmful in some cases by mediating atherosclerotic and proinflammatory effects. Levy [24] reported that a variety of hormones, cytokines, and metabolic factors are associated with an increase in AT2R density on the cell surface. Evidence from human cardiomyocytes suggests that Ang II may promote plaque rupture by increasing matrix metalloproteinase-1 in an AT2-dependent manner [32]. Ruiz-Ortega et al [33] demonstrated that 72 hours of Ang II infusion increased the number of glomeruli and interstitial inflammatory cells. In addition, AT4R activation promotes the release of PAI-1 before thrombosis, which may be related to the occurrence of acute coronary syndrome. Thus, ACEIs should be considered as first-line therapy to limit the excess mortality and morbidity in this population.

Can the combination therapy with an ACEI and an ARB provide greater renal and cardiovascular protection to the patients with CKD3-5? Previous guidelines [2014 the Eighth Joint National Committee (JNC 8) guidelines] do not recommend the use of dual blockade with ACEIs and ARBs in non-dialysis CKD3-5 patients [34]. In a study conducted in pre-dialysis stage 5 CKD patients, risk of death in those receiving dual blockade with ACEIs and ARBs was 1.49 times and 1.58 times higher, respectively, than those who received ARB monotherapy in all CKD patients and in diabetic subgroup [35]. A previous meta-analysis showed that although combined RAAS blockade therapy decreased albuminuria and proteinuria in CKD patients, there was no benefit on doubling of the serum creatinine level, or mortality [20]. Our findings showed that dual therapy with an ACE inhibitor and an ARB had no additional beneficial effect on all primary outcomes, compared to ACEI monotherapy, ARB monotherapy and any other treatments. Reversely, it significantly increased the odds of hyperkalaemia, 4.8-times higher than ARB monotherapy and 6.43-times higher than ACEI monotherapy. However, Palmer et al [18] reported a meta-analysis of 157 RCTs in patients with diabetic kidney disease that showed ACEI+ARB as superior to other antihypertensive agents including monotherapy in regressing albuminuria, and preventing progression to ESKD. This inconsistency may be because our analysis on combination of ACEI + ARB was mainly driven by ONTARGET study with a large sample size (5623 patients), a new study that was included in our study, that was not considered in the previous meta-analyses. Besides, the meta-analysis conducted by Palmer et al [18] focused on DKD patients, but not CKD/DKD patients with eGFR $<60 \mathrm{~mL} / \mathrm{min} / 1.73 \mathrm{~m}^{2}$. Thus, the effectiveness of dual RAS blockade in CKD3-5 patients with eGFR $<60 \mathrm{~mL} / \mathrm{min} / 1.73 \mathrm{~m}^{2}$ may not be valid. Based on at least current findings, dual RAS blockade is not recommended for this population.

The safety of RAS inhibitors in CKD patients with reduced GFRs was the focus of concern. Many investigators did not consider RAS inhibitions in a population with severe reduced renal function due to various considerations, including concerns about hyperkalaemia or hypotension, which may occur in this weak population. Previous studies in CKD patients showed therapy with RAAS-blocking agents was associated with higher risk of hyperkalaemia, cough and hypotension [16, 18, 20, 36-38]. Of these side effects, hyperkalaemia was a major concern. Hsu et al performed a study, which enrolled 28,497 patients with serum creatinine levels $>6 \mathrm{mg} / \mathrm{dL}$, published in JAMA Intern Med. Results indicated that ACEI/ARB users (9.2\%) had a higher hyperkalaemia-associated hospitalisation rate than non-users (6.7\%), but those who developed hyperkalaemia did not significantly increase the risk of pre-dialysis death [37]. These results were generally in accordance with our results. In the present meta-analysis, ACEI/ARB monotherapy had higher odds of hyperkalaemia. Besides, we also found ACEI/ARB combination therapy had the worst safety with a significantly increased risk of hyperkalaemia compared to all treatments. In 2028, NKF-KDOQI suggested hyperkalaemia prevention and management protocols, including dietary potassium restriction, correction of acid-base imbalances, avoidance of concomitant use of medications that promote hyperkalaemia, optimisation of diuretic use, and use of cation-exchange resins, may be used to prevent hyperkalaemia when clinically indicated, thus helpful to balance the effects and safety of RAAS blockades [39].

To date, there is little information available with regard to patients with CKD3-5 and diabetes mellitus. A previous pair-wise meta-analysis including nine trials, conducted in non-dialysis diabetic patients ( $n=9797$ participants) with CKD stages 3-5, found that RAAS-blocking agents could lower the risk of renal replacement therapy/doubling of serum creatinine, but had no beneficial effect on all-cause mortality and cardiovascular mortality events compared to placebo or other antihypertensive agents [15]. However, most of these effects were attributed to only three trials 
which used a head-to-head comparison between these two types of agents [15]. According to our subgroup analyses for patients with diabetes, only ARBs had significant superiority over placebo in preventing the occurrence of kidney events, ranking in the top position with highest SUCRA probability. No drugs, including ACEIs or ARBs, significantly lowered the risk of cardiovascular events and all-cause death compared to placebo, consistent with previous findings. Even so, in our study, ACEIs still had the strongest protective effect in all-cause death according to SUCRA. A previous metaanalysis conducted in patients with diabetes and albuminuria also showed ARBs reduced risks of ESRD and doubled the serum creatinine level, and both ACEIs and ARBs failed to reduce all-cause mortality and CV [40]. Why did ACEI and ARB produce different effects in patients with diabetic nephropathy and non-diabetic nephropathy? There are no studies conducted, as yet, to compare these effects in these two populations. We speculate the adequacy of glycaemic control is an important potential confounding factor in assessing a direct effect of ACEI or ARB on the progression of renal disease in patients with diabetic nephropathy. A favourable effect of glycaemic control may be most important in the very early phases of diabetic nephropathy. Besides, our result was mainly contributed by direct comparisons in only one study-PRONEDI 2013 [41] and indirect comparisons among other seven studies [42-48]. Thus, the absence of a positive effect on renal and cardiovascular events and mortality by ACEIs in our meta-analysis should not be considered as conflictive with the general literature. We believe that, in the future, when more and larger studies are included, the advantages of ACEIs in improving survival of patients with advanced diabetic nephropathy will be more prominent.

In CKD patients with serum creatinine $>200 \mu \mathrm{mol} / \mathrm{L}$, ACEIs and ARBs were the two most effective drugs in all primary outcomes with worse ranks of hyperkalaemia. A meta-analysis conducted in 20,152 CKD patients with serum creatinine $>6 \mathrm{mg} / \mathrm{dL}$ also drew a similar result that the use of ACEIs/ARBs was associated lower risk of long-term dialysis or death by $6 \%$ and higher risk of hyperkalaemia-associated hospitalisations than among non-users $(9.2 \%$ vs $6.7 \%$; $p<0.001$ ), making our conclusion more convincing [37]. When sample size was larger than 100 and follow-up longer than three years, ACEIs had lower risk of all primary outcomes than placebo, and still had the greatest probability to be the best treatment option for CKD3-5 patients, consistent with the results in standard analyses. Notably, in studies with sample size larger than 100, ACEIs and $\beta$-blockers involved significantly increased risk of hyperkalaemia compared to placebo, which was something different. Nevertheless, the identification of sub-groups of patients who may benefit is important to help guide treatment and should be explored in future studies.
The large volume of included data and rigorous statistic methodology were the strengths of this meta-analysis. However, our study had some limitations. First, the emphasis of this study was the effects of RAS inhibitors on kidney or cardiovascular protection. Trials with comparisons among other antihypertensive agents or placebo were not included, which limits the strength of the conclusions of the network analysis. Second, we did not analyse the influences of blood pressure reduction and risk of AKI among different antihypertensive treatments because of poorly available data. Third, scant primary data also prevented us from undertaking subgroup analysis of the primary outcomes of treatments by different CKD stages, leaving effects of blood pressure treatment on cardiovascular events and mortality in this population uncertain. Finally, we conducted a protocol before performing our research, but it has not been submitted for publication, which does not meet the PRISMA criteria for a published protocol.

\section{Conclusion}

The findings of our network meta-analysis suggested that although RAS blockade therapy had a definite increase in the odds of hyperkalaemia, hypotension, and cough in patients with non-dialysis CKD3-5, it was still more beneficial to this population. ACEIs, superior to ARBs and other antihypertensive drugs, had the highest probability of being the most beneficial treatments for kidney events, cardiovascular outcomes and all-cause mortality in non-dialysis CKD3-5 patients. In the subgroup of patients with diabetic kidney disease, ARBs were superior to ACEIs in lowering probability of kidney events and cardiovascular events but fell behind ACEIs in all-cause death. Even so, we still have to consider the potential harms of these treatments. Physicians need to balance the benefits and potential harms of these treatments when selecting the preparation and dose for individual patients. The utilisation of hyperkalaemia prevention and management protocols would help to balance serum potassium levels, making it possible to initiate or continue the use of RAS inhibition among patients with CKD3-5 in the future.

\section{Compliance with Ethical Standards}

Funding This work was supported by grants from the National Natural Science Foundation $(81600553,81270791$, and 30800529), Tianjin Municipal Science and Technology Commission Foundation (14JCYBJC27900) and Tianjin Public Health Bureau Foundation (2014KR16), The 12th Five-Year Plan National Science and Technology Support Program (No. 2011BAI10B02) and Shanxi Public Health Bureau Foundation (201302051), the General Hospital of Tianjin Medical University Youth Incubation Foundation (ZYYFY2015001). 
Conflict of interest Yaru Zhang, Dandan He, Wei Zhang, Yue Xing, Yipeng Guo, Fuzhen Wang, Junya Jia, Tiekun Yan, Youxia Liu and Shan Lin declare no conflict of interest.

Open Access This article is licensed under a Creative Commons Attribution-NonCommercial 4.0 International License, which permits any non-commercial use, sharing, adaptation, distribution and reproduction in any medium or format, as long as you give appropriate credit to the original author(s) and the source, provide a link to the Creative Commons licence, and indicate if changes were made. The images or other third party material in this article are included in the article's Creative Commons licence, unless indicated otherwise in a credit line to the material. If material is not included in the article's Creative Commons licence and your intended use is not permitted by statutory regulation or exceeds the permitted use, you will need to obtain permission directly from the copyright holder.To view a copy of this licence, visit http://creativecommons.org/licenses/by-nc/4.0/.

\section{References}

1. Hill NR, Fatoba ST, Oke JL, et al. Global prevalence of chronic kidney disease - a systematic review and meta-analysis. PLoS One. 2016;11(7):e0158765.

2. Weiner DE, Tighiouart H, Amin MG, et al. Chronic kidney disease as a risk factor for cardiovascular disease and all-cause mortality: a pooled analysis of community-based studies. J Am Soc Nephrol. 2004;15(5):1307-15.

3. Tonelli M, Wiebe N, Culleton B, et al. Chronic kidney disease and mortality risk: a systematic review. J Am Soc Nephrol. 2006;17(7):2034-47.

4. Mahmoodi BK, Matsushita K, Woodward M, et al. Associations of kidney disease measures with mortality and end-stage renal disease in individuals with and without hypertension: a metaanalysis. Lancet. 2012;380(9854):1649-61.

5. Go AS, Chertow GM, Fan D, McCulloch CE, Hsu CY. Chronic kidney disease and the risks of death, cardiovascular events, and hospitalization. N Engl J Med. 2004;351(13):1296-305.

6. de Goeij MC, Voormolen N, Halbesma N, et al. Association of blood pressure with decline in renal function and time until the start of renal replacement therapy in pre-dialysis patients: a cohort study. BMC Nephrol. 2011;12:38.

7. Ettehad D, Emdin CA, Kiran A, et al. Blood pressure lowering for prevention of cardiovascular disease and death: a systematic review and meta-analysis. Lancet. 2016;387(10022):957-67.

8. Herzog CA, Asinger RW, Berger AK, et al. Cardiovascular disease in chronic kidney disease. A clinical update from Kidney Disease: Improving Global Outcomes (KDIGO). Kidney Int. 2011;80(6):572-86.

9. Xie X, Liu Y, Perkovic V, et al. Renin-Angiotensin system inhibitors and kidney and cardiovascular outcomes in patients with CKD: a Bayesian network meta-analysis of randomised clinical trials. Am J Kidney Dis. 2016;67(5):728-41.

10. Taler SJ, Agarwal R, Bakris GL, et al. KDOQI US commentary on the 2012 KDIGO clinical practice guideline for management of blood pressure in CKD. Am J Kidney Dis. 2013;62(2):201-13.

11. Lumley T. Network meta-analysis for indirect treatment comparisons. Stat Med. 2002;21(16):2313-24.

12. Tu YK, Needleman I, Chambrone L, Lu HK, Faggion CM. A Bayesian network meta-analysis on comparisons of enamel matrix derivatives, guided tissue regeneration and their combination therapies. J Clin Periodontol. 2012;39(3):303-14.
13. Salanti G, Higgins JP, Ades A, Ioannidis JP. Evaluation of networks of randomised trials. Stat Methods Med Res. 2007;17(3):279-301.

14. Fried LF, Emanuele N, Zhang JH, et al. Combined angiotensin inhibition for the treatment of diabetic nephropathy. N Engl J Med. 2013;369(20):1892-903.

15. Nistor I, De Sutter J, Drechsler C, et al. Effect of renin-angiotensin-aldosterone system blockade in adults with diabetes mellitus and advanced chronic kidney disease not on dialysis: a systematic review and meta-analysis. Nephrol Dial Transplant. 2018;33(1):12-22.

16. Ren F, Tang L, Cai Y, et al. Meta-analysis: the efficacy and safety of combined treatment with ARB and ACEI on diabetic nephropathy. Ren Fail. 2015;37(4):548-61.

17. Wu HY, Huang JW, Lin HJ, et al. Comparative effectiveness of renin-angiotensin system blockers and other antihypertensive drugs in patients with diabetes: systematic review and bayesian network meta-analysis. BMJ. 2013;347:f6008.

18. Palmer SC, Mavridis D, Navarese E, et al. Comparative efficacy and safety of blood pressure-lowering agents in adults with diabetes and kidney disease: a network meta-analysis. Lancet. 2015;385(9982):2047-56.

19. Balamuthusamy S, Srinivasan L, Verma M, et al. Renin angiotensin system blockade and cardiovascular outcomes in patients with chronic kidney disease and proteinuria: a meta-analysis. Am Heart J. 2008;155(5):791-805.

20. Susantitaphong P, Sewaralthahab K, Balk EM, Eiam-ong S, Madias NE, Jaber BL. Efficacy and safety of combined vs. single renin-angiotensin-aldosterone system blockade in chronic kidney disease: a meta-analysis. Am J Hypertens. 2013;26(3):424-41.

21. Qin Y, Chen T, Chen Q, et al. The effect of angiotensin-converting enzyme inhibitor/angiotensin receptor blocker use on mortality in patients with chronic kidney disease: a meta-analysis of observational studies. Pharmacoepidemiol Drug Saf. 2016;25(5):503-11.

22. Campbell DJ, Kladis A, Duncan AM. Effects of converting enzyme inhibitors on angiotensin and bradykinin peptides. Hypertension. 1994;23(4):439-49. https://doi.org/10.1161/01. hyp.23.4.439 (PMID: 8144213).

23. Zaman MA, Oparil S, Calhoun DA. Drugs targeting the renin-angiotensin-aldosterone system. Nat Rev Drug Discov. 2002;1(8):621-36.

24. Levy BI. Can angiotensin II type 2 receptors have deleterious effects in cardiovascular disease? Implications for therapeutic blockade of the renin-angiotensin system. Circulation. 2004;109(1):8-13.

25. Mogi M, Iwai M, Horiuchi M. Emerging concept of adipogenesis regulation by the renin-angiotensin system. Hypertension. 2006;48(6):1020-2. https://doi.org/10.1161/01.HYP.0000248196 .14826.31 (PMID: 17060513).

26. Siragy HM, de Gasparo M, Carey RM. Angiotensin type 2 receptor mediates Valsartan-Induced hypotension in conscious rats. Hypertension. 2000;35(5):1074-7.

27. Volpe M, Tocci G, Savoia C. Angiotensin II receptor blockers and coronary artery disease: 'presumed innocents'. 2006;27(12):1506.

28. Siragy HM, Carey RM. The subtype-2 (AT2) angiotensin receptor regulates renal cyclic guanosine 3',5'-monophosphate and AT1 receptor-mediated prostaglandin $\mathrm{E} 2$ production in conscious rats. J Clin Invest. 1996;97(8):1978.

29. Siragy HM, Jaffa AA, Margolius HS, Carey RM. Reninangiotensin system modulates renal bradykinin production. 1996;271(2):1090-5.

30. Mehta PK, Griendling KK. Angiotensin II cell signaling: physiological and pathological effects in the cardiovascular system. Am J Physiol Cell Physiol. 2007;292(1):C82.

31. Carey RM. Cardiovascular and renal regulation by the angiotensin type 2 receptor: the AT2 receptor comes of age. Hypertension. 
2005;45(5):840-4. https://doi.org/10.1161/01.HYP.0000159192 .93968.8f (PMID: 15738342).

32. Kim MP. Angiotensin II increases human monocyte matrix metalloproteinase-1 through the AT2 receptor and prostaglandin E2: implications for atherosclerotic plaque rupture. J Leukoc Biol. 2005;78(1):195-201.

33. Ruiz-Ortega M, Lorenzo Ó, Rupérez M, Blanco J, Egido J. Systemic infusion of angiotensin II into normal rats activates nuclear factor- $\mathrm{\kappa B}$ and AP-1 in the kidney. Am J Pathol. 2001;158(5):1743-56.

34. James PA, Oparil S, Carter BL, et al. 2014 evidence-based guideline for the management of high blood pressure in adults: report from the panel members appointed to the Eighth Joint National Committee (JNC 8). JAMA. 2014;311(5):507-20.

35. Lin CC, Wu YT, Yang WC, et al. Angiotensin receptor blockers are associated with lower mortality than ACE inhibitors in predialytic stage 5 chronic kidney disease: a nationwide study of therapy with renin-angiotensin system blockade. PLoS One. 2017;12(12):e0189126.

36. Bandak G, Sang Y, Gasparini A, et al. Hyperkalemia after initiating renin-angiotensin system blockade: the stockholm creatinine measurements (SCREAM) project. J Am Heart Assoc. 2017;6(7): 0005428.

37. Hsu TW, Liu JS, Hung SC, et al. Renoprotective effect of reninangiotensin-aldosterone system blockade in patients with predialysis advanced chronic kidney disease, hypertension, and anemia. JAMA Intern Med. 2014;174(3):347-54.

38. Weinberg JM, Appel LJ, Bakris G, et al. Risk of hyperkalemia in nondiabetic patients with chronic kidney disease receiving antihypertensive therapy. Arch Intern Med. 2009;169(17):1587-94.

39. Weir MR, Lakkis JI, Jaar B, et al. Use of renin-angiotensin system blockade in advanced CKD: an NKF-KDOQI controversies report. Am J Kidney Dis. 2018;72:873-84.
40. Wang K, Hu J, Luo T, Wang Y, Yang S, Qing H, Cheng Q, Li Q. Effects of angiotensin-converting enzyme inhibitors and angiotensin II receptor blockers on all-cause mortality and renal outcomes in patients with diabetes and albuminuria: a systematic review and meta-analysis. Kidney Blood Press Res. 2018;43:768-79.

41. Fernandez JG, Luño J, Barrio V, et al. Effect of dual blockade of the renin-angiotensin system on the progression of type 2 diabetic nephropathy: a randomised trial. Am J Kidney Dis. 2013;61(2):211-8.

42. Imai E, Chan JC, Ito $S$, et al. Effects of olmesartan on renal and cardiovascular outcomes in type 2 diabetes with overt nephropathy: a multicentre, randomised, placebo-controlled study. Diabetologia. 2011;54(12):2978-86.

43. Berl T, Hunsicker LG, Lewis JB, et al. Cardiovascular outcomes in the Irbesartan Diabetic Nephropathy Trial of patients with type 2 diabetes and overt nephropathy. Ann Intern Med. 2003;138(7):542-9.

44. Brenner BM, Cooper ME, de Zeeuw D, et al. Effects of losartan on renal and cardiovascular outcomes in patients with type 2 diabetes and nephropathy. N Engl J Med. 2001;345(12):861-9.

45. Tong PC, Ko GT, Chan WB, et al. The efficacy and tolerability of fosinopril in Chinese type 2 diabetic patients with moderate renal insufficiency. Diabetes Obes Metab. 2006;8(3):342-7.

46. Bakris GL, Copley JB, Vicknair N, Sadler R, Leurgans S. Calcium channel blockers versus other antihypertensive therapies on progression of NIDDM associated nephropathy. Kidney Int. 1996;50(5):1641-50.

47. Fogari R, Zoppi A, Corradi L, et al. Long-term effects of ramipril and nitrendipine on albuminuria in hypertensive patients with type II diabetes and impaired renal function. J Hum Hypertens. 1999;13(1):47-53.

48. Sawicki PT, Berger M. Renal protective effect of enalapril in diabetic nephropathy. BMJ. 1992;304(6830):841 (author reply 841-2).

\section{Affiliations}

\section{Yaru Zhang ${ }^{1}$ - Dandan $\mathrm{He}^{1} \cdot$ Wei Zhang ${ }^{2} \cdot$ Yue Xing ${ }^{1} \cdot$ Yipeng Guo ${ }^{3} \cdot$ Fuzhen Wang $^{4}$ - Junya Jia ${ }^{1} \cdot$ Tiekun Yan $^{1}$. Youxia Liu ${ }^{1}$. Shan Lin ${ }^{1}$}

Junya Jia

jiajunya@126.com

Youxia Liu

5liuyouxia@163.com

1 Department of Nephrology, General Hospital of Tianjin Medical University, No. 154, Anshan road, Heping district, Tianjin, China
2 Department of Cardiac Surgery, Tianjin Chest Hospital, Tianjin, China

3 Department of Epidemiology, Tianjin Public Health Bureau, Tianjin, China

4 Department of Statistics, Fenyang Hospital of Shanxi Province, Fenyang, China 